\title{
Characterization of microlenses by digital holographic microscopy
}

\author{
Florian Charrière, Jonas Kühn, Tristan Colomb, Frédéric Montfort, Etienne Cuche, \\ Yves Emery, Kenneth Weible, Pierre Marquet, and Christian Depeursinge
}

\begin{abstract}
We demonstrate the use of digital holographic microscopy (DHM) as a metrological tool in micro-optics testing. Measurement principles are compared with those performed with Twyman-Green, MachZehnder, and white-light interferometers. Measurements performed on refractive microlenses with reflection DHM are compared with measurements performed with standard interferometers. Key features of DHM such as digital focusing, measurement of shape differences with respect to a perfect model, surface roughness measurements, and optical performance evaluation are discussed. The capability of imaging nonspherical lenses without any modification of the optomechanical setup is a key advantage of DHM compared with conventional measurement tools and is demonstrated on a cylindrical microlens and a square lens array. (C) 2006 Optical Society of America
\end{abstract}

OCIS codes: $\quad 090.0090,090.1760,120.3620$.

\section{Introduction}

Since its principle was proposed by Goodman and Lawrence ${ }^{1}$ and by Kronrod et al. ${ }^{2}$ more than 30 years ago, digital holographic microscopy (DHM) has been developed for a wide range of applications. In particular, off-axis DHM allows the extraction of both amplitude and phase information for a wave diffracted by a specimen from a single hologram. ${ }^{3,4}$ The phase information provides $3 \mathrm{D}$ quantitative mapping of the phase shift induced by microscopic specimens with a resolution along the optical axis (axial resolution) better than $1^{\circ}$. Thanks to the performance of personal computers and progress in digital image acquisition, DHM currently provides cost-effective and easy-touse instruments with high acquisition rates (camera limited) for real-time measurements and quality control in production facilities.

F. Charrière (florian.charriere@a3.epfl.ch), J. Kühn, T. Colomb, F. Monfort, and C. Depeursinge are with the Ecole Polytechnique Fédérale de Lausanne, Imaging and Applied Optics Institute, CH1015 Lausanne, Switzerland. E. Cuche and Y. Emery are with Lyncée Tec SA, PSE-A, CH-1015 Lausanne, Switzerland. K. Weible is with SUSS MicroOptics SA, Jacquet-Droz 7, CH-2007 Neuchâtel, Switzerland. P. Marquet is with the Centre de Neurosciences Psychiatriques, Département de Psychiatrie, DP-CHUV, Site de Cery, CH-1008 Prilly-Lausanne, Switzerland.

Received 13 May 2005; revised 8 August 2005; accepted 8 August 2005.

0003-6935/06/050829-07\$15.00/0

(C) 2006 Optical Society of America
In a general context, DHM technology has been successfully applied for numerous operating modes, for instance, tomography on a biological sample performed with wavelength scanning, 5 investigation of the polarization state of an object by use of two orthogonally polarized wavefronts, ${ }^{6}$ and multiplewavelength interferometry of dynamic systems. ${ }^{7}$

This paper investigates some of the possibilities offered by DHM for micro-optics quality control, a domain with high performance demands in terms of speed, precision, automation, and productivity. The results are compared with those performed with Twyman-Green, Mach-Zehnder, and white-light interferometers (WLIs). Kebbel et al. ${ }^{8}$ demonstrated measurement on a cylindrical lens with a dualwavelength lensless DHM configuration, requiring averaging of 15 frames. This system achieved an axial resolution of $283 \mathrm{~nm}$. We demonstrate off-axis DHM measurements requiring a single hologram acquisition and yielding an axial precision of $3.7 \mathrm{~nm}$ $(\lambda / 175)$ for measurement of silicon lenses in reflection configuration and $15.8 \mathrm{~nm}(\lambda / 40)$ for quartz lenses in transmission configuration, i.e., at least as good as other conventional interferometers.

\section{Metrology of Microlens Arrays-State of the Art}

The constraints imposed on microlens arrays can be extremely demanding. Precise control of the shape, surface quality, and optical performance of the microlenses are required, as well as uniformity of these parameters across the array. Many different metrol- 
ogy approaches exist, and noncontact techniques are preferred.9,10 The Twyman-Green interferometer is probably the most precise tool for shape characterization, and direct analysis of optical performance is often performed with Mach-Zehnder interferometers. To achieve high precision, these types of interferometer require complex manipulations and optimization procedures that are often difficult to implement as an automated process of quality control, especially for entire wafers. WLIs with fully automated measurement capabilities are commercially available. However, these instruments are often not suited for characterization of the entire lens profile, yielding accurate information for only the vertex of refractive microlenses.

To be able to precisely control and optimize the performance of refractive microlens arrays, all the standard metrology tools mentioned above can be exploited with each giving a partial view of the total picture. It is only by combining the information from all these measurements that the tight constraints imposed on microlens arrays can be controlled and maintained. The specificities of the various types of interferometers are described hereafter.

Twyman-Green interferometers seem to be the most accurate tools for characterizing the shape of spherical or weakly aspherical lenses. Measurements in reflection provide the deviation of the surface shape from an ideal sphere, with an axial resolution in the range of $\lambda / 20$. A piezoelectric transducer is used to shift the reference mirror to introduce a phase shift of $\pi / 2$ between individual measurements. A phase-shifting algorithm is then used to calculate the reflected wavefront. ${ }^{11}$ The measured unwrapped phase is used to make a direct comparison of the measured and the desired lens profiles and also permits the measurement of the radius of curvature (ROC) of the lens. Strong aspherical and nonspherical (e.g., cylindrical) lenses cannot be characterized in this way. The instrument used for this paper has been described in Ref. 12. One has to note that, in practice, ROC measurement with a Twyman-Green interferometer is performed by fringe analysis with the lens in a cat's eye configuration, i.e., when the focal point of the microscope objective coincides with the vertex of the microlens. In this position, the fringes of the hologram are perfectly straight lines. The lens is then mechanically moved until the fringes disappear, i.e., when the ROC of the lens matches the ROC of the illuminating wavefront; the vertical displacement of the microlens from the cat's eye position to the standard measurement position corresponds to the ROC of the microlens. ROC measured this way depends on the mechanical stage precision and remains in the micrometer precision range.

Mach-Zehnder interferometers allow for direct analysis of the optical performance of the microlenses. Transmission Mach-Zehnder measurements allow the aberrations of microlenses to be determined. The interferometer model used for this paper has been described in Ref. 12. An illumination microscope objective of high quality is used to generate a spherical wavefront, which is then collimated by the microlens under test. The interference of this wavefront with a plane reference wavefront allows for the determination of the optical quality of the microlens and yields an accuracy in the range of $\lambda / 20$. Adapting the optomechanical setup allows Mach-Zehnder interferometers to also be used for the characterization nonspherical microlenses. For cylindrical lenses for instance, the microscope objective that is used to produce the spherical beam is removed. Consequently, a collimated beam impinges on the microlens and the interference pattern describes the phase delay introduced by the microlens. The phase is recovered by a standard phase-shifting technique and unwrapping algorithm. This phase delay can be used to calculate the lens profile that must have produced it. In this way, cylindrical lens profiles can be compared to the theoretically desired lens shape.

The WLI used for comparison in this paper is a WYKO NT3300. It is built in a reflection configuration and is capable of scanning over a complete $200 \mathrm{~mm} \times 200 \mathrm{~mm}$ field. High-precision measurements with subnanometer resolution can be obtained when using the phase-shifting interferometric (PSI) mode, which requires a narrowband filter to produce a nearly monochromatic illumination light source. However, when using the PSI mode, the total depth that can be measured is limited. The entire lens surface may be measured for only small-NA lenses, but in general only the vertex area of the lens may be measured with the PSI mode. This is useful for obtaining the ROC of the best-fit sphere at that vertex of the lens but does not provide substantial information about the full lens shape.

The measurement time is mostly determined by the autofocus routine based on the fringe visibility detection and varies from 2 to $6 \mathrm{~s}$ depending on the amount of light reflected. The autofocus also allows for positioning correction along the optical axis during transverse measurement scanning of microcomponents. Therefore it is important that the sample is correctly disposed, so that the height error due to an incorrect tilt adjustment remains in the autofocus range along the entire sample.

Another application of the WLI is to use the vertical scanning interferometry (VSI) mode to perform a noncontact measurement of the lens height. Information is normally lost at the edge of the lenses due to the steep profile, but the height information at the vertex and at the surrounding substrate can be used to determine the lens height without any contact of the lens surface. VSI measurements are performed with the white-light source without the narrowband filter. Phase shifting is not performed. Instead, the optical head is scanned vertically while digital signal processing is performed to determine the peak of the visibility of the white-light fringes for each pixel in the detector array. The result is a mapping of the height of the imaged surface with a nanometer resolution. This mode is not used in standard micro-optic control.

In summary, reflection Twymann-Green mea- 
(a)

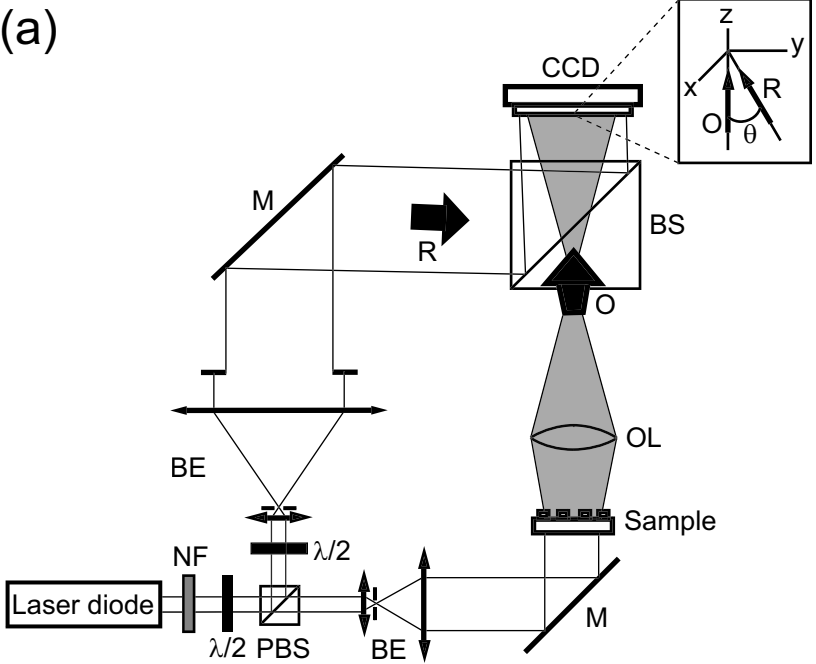

(b)

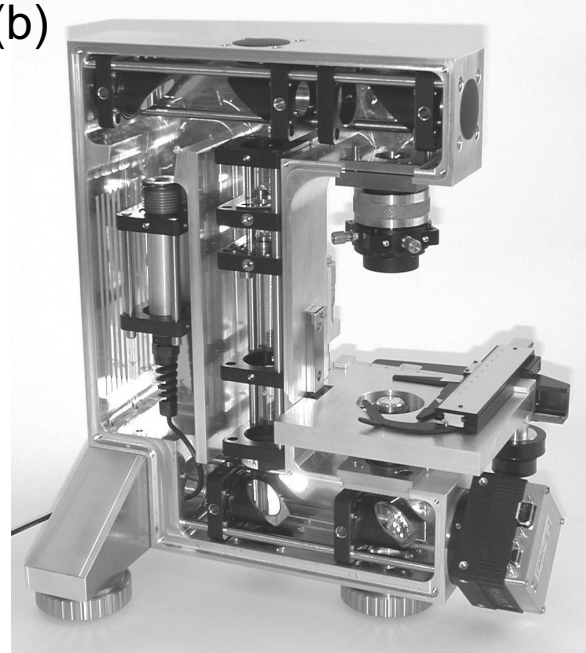

Fig. 1. Holographic microscope for transmission imaging: (a) experimental setup and (b) integrated instrument. NF, neutral-density filter; PBS, polarizing beam splitter; BE, beam expander with spatial filter; $\lambda / 2$, half-wave plate; OL, objective lens; M, mirror; BS, beam splitter; O, object wave; R, reference wave. Inset, detail showing the off-axis geometry at incidence on the CCD.

surements allow precise characterization of the full lens shape, Mach-Zender measurements provide lens aberrations, WLI measurements in the PSI mode provide the ROC and deviation from the best-fit sphere for the vertex of the lens within less than $1 \%$ resolution, and WLI measurements in the VSI mode provide the lens height. A common drawback of all these techniques is the use of PSI phase measurement procedures that are highly sensitive to external perturbations and that require the use of piezoelectric-transducer-controlled moving parts to modulate the phase between successive acquisitions. This results in a relatively low measurement rate between 2 and $6 \mathrm{~s}$ per lens, mainly due to the autofocus procedure, and implementation costs owing to the need of vibration-insulating devices. Moreover, all these techniques require accurate control of the specimen position and orientation, which make them difficult to implement for automated quality control applications.

\section{Digital Holographic Microscopy}

\section{A. Experimental Setup}

The transmission (Fig. 1) and reflection (Fig. 2) DHM used for the present study are described in detail in Ref. 4 . The results presented here were obtained with an objective lens of $8.00 \mathrm{~mm}$ focal length with a NA of 0.50 defining $\times 20$ magnification. As a light source, we used a circular laser diode module with a wavelength of $635 \mathrm{~nm}$. The camera is a standard $512 \times 512$ pixel, 8 bit, black-and-white CCD with a pixel size of $6.7 \mu \mathrm{m} \times 6.7 \mu \mathrm{m}$ and a maximum frame rate of $25 \mathrm{~Hz}$. Both instruments used for this paper have a transverse resolution around $1 \mu \mathrm{m}$. The field of view is $250 \mu \mathrm{m} \times 250 \mu \mathrm{m}$ for the transmission setup and $300 \mu \mathrm{m} \times 300 \mu \mathrm{m}$ for the reflection setup. The transverse resolution, as well as the transverse scale calibration, is determined with the help of a USAF 1951 resolution test target. Note that the transverse resolution, as well as the field of view (FOV) of DHM, can be easily adapted to different specimen sizes, as long as the lateral resolution remains sufficient to properly unwrap the modulo- $2 \pi$ reconstructed phase distribution.

Measurements presented here have been conducted without any system for insulating against vibrations of the building. This is possible thanks to the remarkably high measurement stability and robustness of DHM, which results from the fact that the off-axis configuration allows all the necessary information to be recorded with a single image acquisition of very short duration. The camera used here comprises an electronic shutter, which allows the exposure time to be reduced to $20 \mu \mathrm{s}$. With a $2.8 \mathrm{Ghz}$ Pentium 4 processor the 3D phase reconstruction rate, described in the next chapter, is 15 frames/s, which is what makes DHM ideal for systematic investigations on large volumes of full wafers of microoptic samples.

\section{B. Hologram Reconstruction}

The procedure for hologram processing, in particular for phase reconstruction, is described in detail in Refs. 4, 13, and 14. For the sake of completeness, a short summary is given here. Holograms acquired by the CCD are first submitted to a procedure of apodization ${ }^{14}$ and filtered in the Fourier plane to remove the zeroth-order and the twin image. ${ }^{13}$ Then, the resulting hologram $I_{H}$, is multiplied by a digital reference wave $R_{D}$ that simulates an illumination wave, ${ }^{4}$ and a propagation calculation in the Fresnel approximation is applied to reconstruct a focused image of the specimen in a plane of coordinates $0 \xi \eta$, where a digital phase mask $\Phi(m, n)$ is applied to compensate for the wavefront curvature induced by the objective lens (see Ref. 4). In summary, the reconstructed wavefront $\Psi(m \Delta \xi, n \Delta \eta)$ is computed according to the 

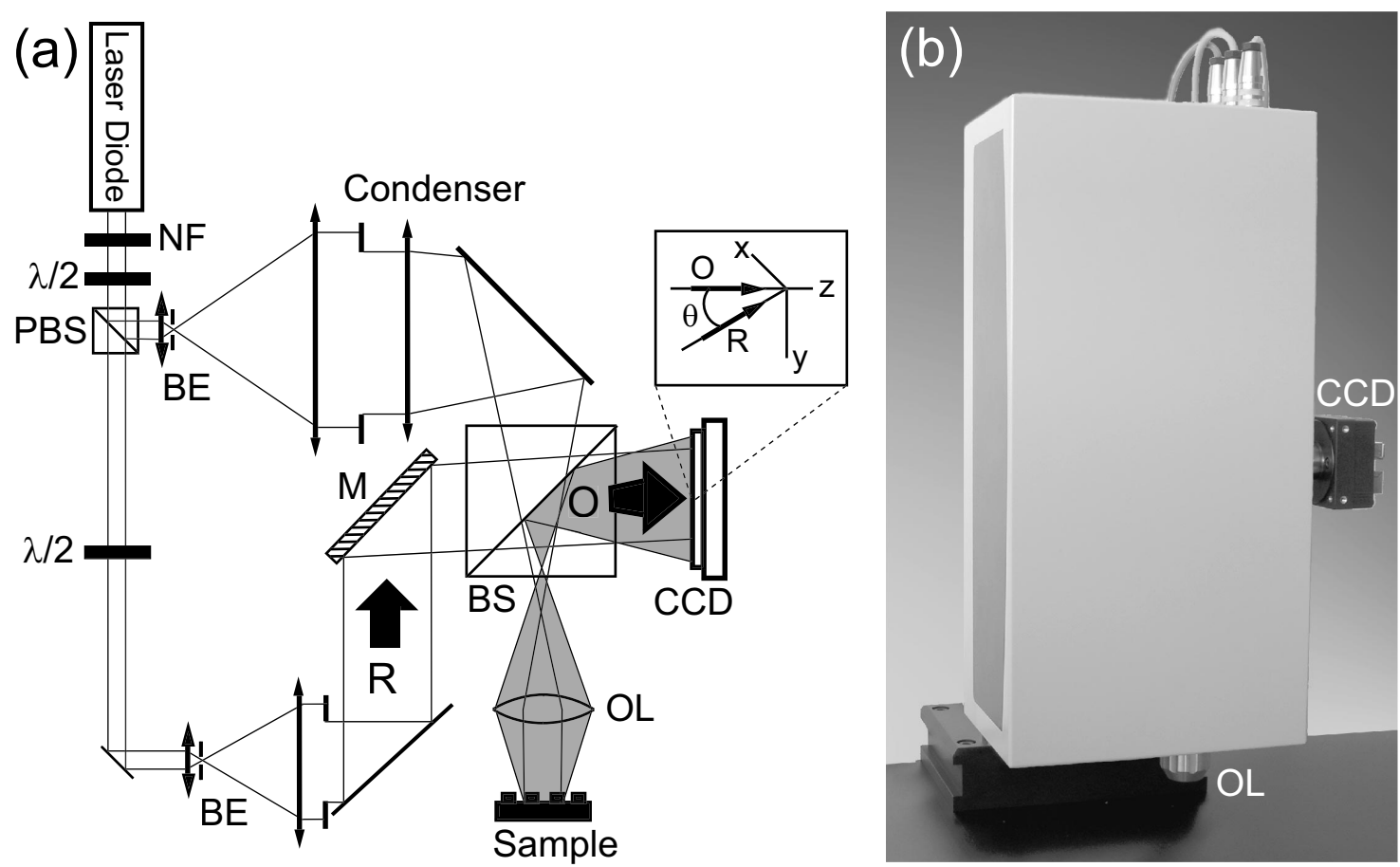

Fig. 2. Holographic microscope for reflection imaging: (a) experimental setup and (b) integrated instrument. Inset, detail showing the off-axis geometry at incidence on the CCD.

following expression:

$$
\begin{aligned}
\Psi(m \Delta \xi, n \Delta \eta)= & A \Phi(m, n) \exp \left[\frac{i \pi}{\lambda d}\left(m^{2} \Delta \xi^{2}+n^{2} \Delta \eta^{2}\right)\right] \\
& \times \operatorname{FFT}\left\{\mathbf{R}_{D}(k, l) I_{H}(k, l)\right. \\
& \left.\times \exp \left[\frac{i \pi}{\lambda d}\left(k^{2} \bar{x}^{2}+l^{2} \bar{y}^{2}\right)\right]\right\}_{m, n}
\end{aligned}
$$

where $m$ and $n$ are integers $(-N / 2 \leq m, n<N / 2)$, FFT is the fast Fourier transform operator, $A=$ $\exp (i 2 \pi d / \lambda) /(i \lambda d), \Delta \xi$ and $\Delta \eta$ are the sampling intervals in the observation plane, $\bar{x}$ and $\bar{y}$ are the pixel size of the CCD, and $k$ and $l$ are integer variables. The digital reference wave is computed using the expression of a plane wave

$$
\mathbf{R}_{D}(k, l)=\exp \left[i\left(k_{D x} \cdot k \bar{x}+k_{D y} \cdot l \bar{y}\right)\right],
$$

where $k_{D x}$ and $k_{D y}$ are the two components of the wave vector. The digital phase mask is computed according to the expression of a parabolic wavefront

$$
\Phi(m, n)=\exp \left[-i \pi /\left(\lambda d_{1}\right) m^{2} \Delta \xi^{2}-i \pi /\left(\lambda d_{2}\right) n^{2} \Delta \eta^{2}\right]
$$

where parameters $d_{1}$ and $d_{2}$ define the field curvature along $0 \xi$ and $0 \eta$, respectively, digitally adjusted to correct the defocusing aberration due to the objective lens. $\Delta \xi$ and $\Delta \eta$ are the sampling intervals in the observation plane.

\section{Parameter Adjustment}

Equation (1) requires the adjustment of four parameters for proper reconstruction of the phase distribution. $k_{D x}$ and $k_{D y}$ compensate for the tilt aberration resulting from the off-axis geometry or resulting from an imperfect orientation of the specimen surface, which should be accurately oriented perpendicular to the optical axis. $d_{1}$ and $d_{2}$ correct the wavefront curvature according to a parabolic model; in principle, these two parameters have similar values, but in the presence of astigmatism it may be that better results can be achieved with slightly different values. As explained in Ref. 4, the parameter values are adjusted to obtain a constant and homogeneous phase distribution on a flat reference surface located in or near the specimen. With microlenses the substrate is ideal to serve as a reference surface. The manual procedure described in Ref. 4 was implemented here as a semiautomated procedure. First, the program extracts two lines-a horizontal line along $0 \xi$ and a vertical line along $0 \eta$-whose locations are defined by the operator in the reference surface. Then, $1 \mathrm{D}$ phase data extracted along the two lines are unwrapped ${ }^{15}$ to remove $2 \pi$ phase jumps, and a curve-fitting procedure is applied to evaluate the unwrapped phase data with a 1D polynomial function of the second order. $k_{D x}$ and $d_{1}$ are iteratively adjusted to minimize the deviation between the fitted curve and the ideal horizontal constant profile. In the same way, $k_{D y}$ and $d_{2}$ are adjusted until the vertical profile is as close as possible to the ideal vertical constant profile. In general, fewer than five iterations are necessary to reach optimal parameter values. If a reference area is not available on the specimen, the parameters are first 

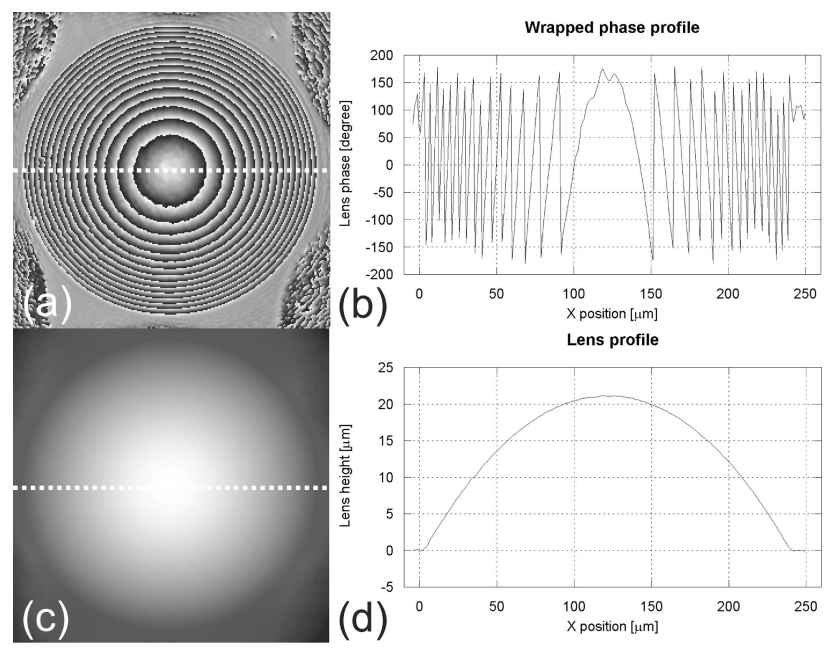

Fig. 3. Phase images of a quartz refractive transmission lens (diameter of $240 \mu \mathrm{m}$, maximal measured height of $21.15 \mu \mathrm{m}$, measured ROC of $351 \mu \mathrm{m}$ ) obtained with transmission DHM: (a) wrapped and (c) unwrapped $2 \mathrm{D}$ representations with corresponding (b) phase and (d) height profiles taken along the two dashed lines in (a) and (c).

calculated on another reference surface (air in transmission, a mirror in reflection); then a simple digital tilt adjustment of the phase, corresponding to an adjustment of $k_{D x}$ and $k_{D y}$, is performed with the same procedure described above when the specimen is observed.

The digital processing of holograms presented in this study is a novel approach in the sense that it performs a numerical reshaping of complex wavefronts and of their propagation, thereby replacing the need for complex optical adjustment procedures. For instance, orienting a mirror or a beam splitter with translation or rotation tables-a task that has to be performed very accurately in classical interferometry - is simply replaced here by the digital adjustment of the wave vector components $k_{D x}$ and $k_{D y}$. Even more relevant is the digital correction of the wavefront deformation induced by the microscope objective. In comparison, in Linnik interference microscopy, the experimental counterpart of adjusting $d$ is achieved by introducing in the reference arm a second identical microscope objective that must be once again aligned with high precision.

\section{Performance for Phase Measurements}

DHM provides quantitative phase mapping. In reflection, the phase information provides the surface topography and permits direct measurement of the lens shape and associated parameters such as the ROC. As for white-light interferometry, reflection measurements are restricted to the vertex of the lens, except if the lens curvature is small enough to ensure proper phase unwrapping up to the lens border. In transmission, the phase information gives the distribution of the optical path length, which describes the phase function of the lens. The geometrical thickness of the lens can then be deduced from the knowledge of its

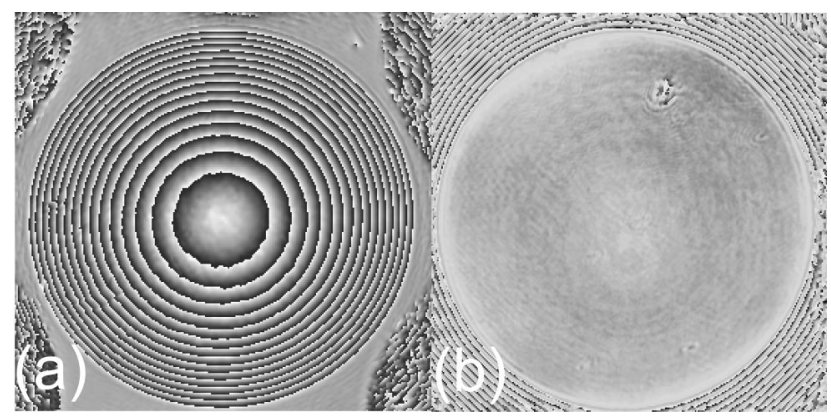

Fig. 4. Comparison between the real and the ideal profile is possible with DHM. By adjusting the reconstruction parameters involved in the reconstruction process, either the wavefront deformations of the objective lens are compensated [standard mode, (a)] or the spherical surface of the lens (here a quartz transmission lens) is compensated (compensation mode). The residue is obtained as the difference between the real and the ideal profile, presented in (b).

refractive index, as well as the lens shape, height, and ROC if the lens has a flat face (e.g., a planoconvex lens).

The precision for phase measurements, which define the axial resolution of DHM, was evaluated with two definitions. The first is measurement of the spatial standard deviation or spatial root-mean-square error (RMSE) over the whole FOV with a flat reference sample. In transmission, with simply ambient air as a reference specimen, the spatial RMSE is $4.1^{\circ}$, which corresponds to a thickness of $15.8 \mathrm{~nm}$ for quartz $(\lambda / 40)$ and to an equivalent height of $3.7 \mathrm{~nm}$ $(\lambda / 175)$ in reflection, where a mirror is used as a reference. The second definition is measurement of the temporal standard deviation, or rms repeatability, for each pixel over 4500 successive measurements during $5 \mathrm{~min}$ and averaged over the whole FOV. Measurements yield $0.46^{\circ}$, corresponding to $1.8 \mathrm{~nm}$ for quartz $(\lambda / 355)$ in transmission and $0.4 \mathrm{~nm}(\lambda / 1565)$ in reflection.

\section{Results: Measurement on Microlenses with Digital Holographic Microscopy}

Three different types of microlenses are analyzed: a quartz refractive transmission lens, a silicon refractive reflective lens, and a cylindrical quartz transmission lens.

Figure 3 presents the measurements of a $240 \mu \mathrm{m}$ diameter quartz spherical lens observed in transmission. Figure 3(a) presents the modulo- $2 \pi$ phase image in 3D perspective, and Fig. 3(c) presents the unwrapped phase image. Two profiles extracted from the center of the lens are presented in Figs. 3(b) and 3(d). Phase unwrapping is performed numerically using a noniterative least-squares algorithm. ${ }^{15}$ In Fig. 3(a), one can observe four disturbed zones at the corners of the image. This corresponds to the four nearest lenses on the array, which are not resolved here because in these regions light deviations occur over large angles that cannot be collected by the objective lens. This also causes the depletion in the 


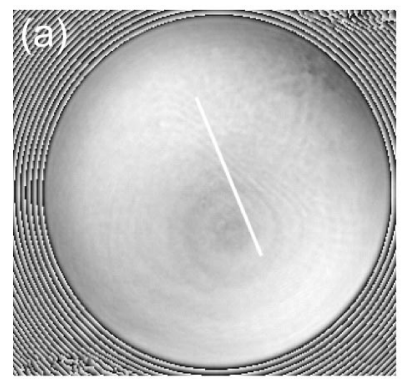

(b)

$$
\begin{aligned}
\mathrm{Ra} & =4.2 \mathrm{~nm} \\
\mathrm{Rt} & =26.7 \mathrm{~nm} \\
\mathrm{Rq} & =5.2 \mathrm{~nm}
\end{aligned}
$$

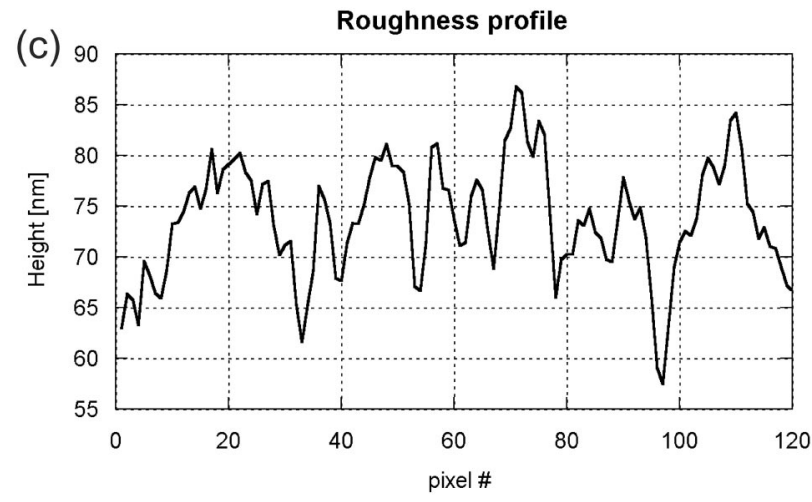

Fig. 5. Roughness measurement on the surface of a silicon refractive reflective lens (diameter of $241 \mu \mathrm{m}$, height of $4.38 \mu \mathrm{m}$ ). A comparison between the real and the ideal profile is possible with DHM. After adjustment of the reconstruction parameters, the residue is obtained as the difference between the real and the ideal profile, presented in (a). Some standard roughness values calculated over a profile are presented in (b), and the extracted profile, corresponding to the white line in (a), is presented in (c).

corners of the unwrapped image in Fig. 3(c) because the unwrapping procedure fails in these zones. The results presented in Fig. 3 allow direct estimation of the lens shape, in particular the ROC and the height.

An interesting feature of DHM is that the digital phase mask [Eq. (3)] involved in the reconstruction process can be defined flexibly by changing the reconstruction parameters $k_{D x}, k_{D y}, d_{1}$, and $d_{2}$. This offers original and efficient possibilities in micro-optics testing since it permits a theoretical model to be fitted to the observed sample and a direct representation of the deviation from this perfect shape to be obtained. The usual parameter adjustment is performed on the phase data extracted from the micro-optical component surface itself instead of calculating it on a flat reference.

Figure 4 illustrates this original feature of DHM with the same quartz lens previously presented. Figure 4(a) shows the phase image obtained by the standard adjustment procedure, and Fig. 4(b) shows the reconstruction of the same hologram when the parameters are adjusted by fitting the phase data on two profiles (one vertical and one horizontal) extracted from the center of the lens. A first advantage of this representation of the lens is that small defects, scratches or material inhomogeneities, become more apparent. The surface quality, roughness, for example, can also be evaluated independently of the lens shape. For example, Fig. 5(a) presents the deviation

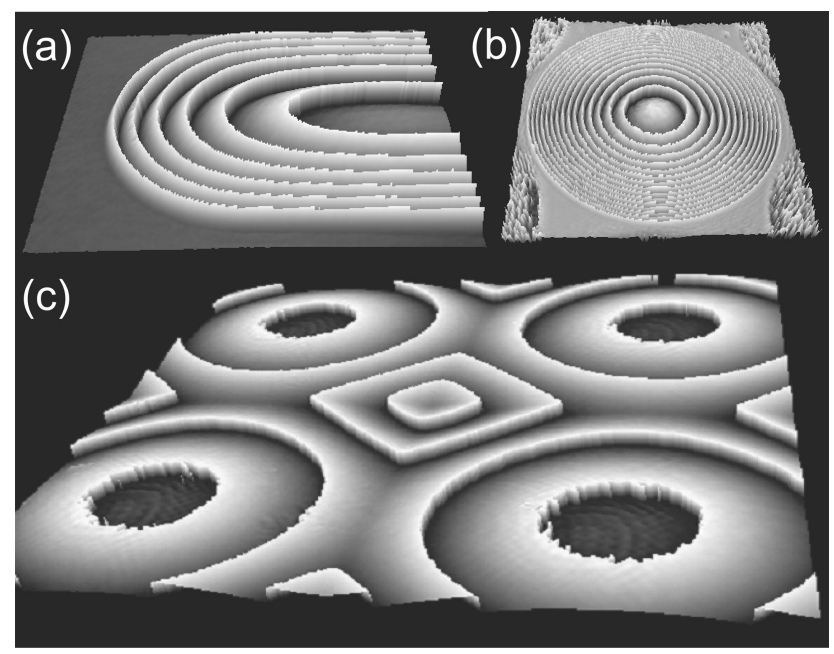

Fig. 6. Perspective phase images of three different lens types measured with the same transmission DHM: (a) cylindrical quartz refractive transmission lens (diameter of $160 \mu \mathrm{m}$, maximal measured height of $7.73 \mu \mathrm{m}$, measured ROC of $417.8 \mu \mathrm{m}$ ), (b) quartz refractive transmission lens (diameter of $240 \mu \mathrm{m}$, maximal measured height of $21.15 \mu \mathrm{m}$, measured ROC of $351 \mu \mathrm{m}$ ), and (c) square quartz lens array (pitch of $500 \mu \mathrm{m}$, maximal height of 5.5 $\mu \mathrm{m}, \mathrm{ROC}$ of $5600 \mu \mathrm{m})$.

from a parabolic surface of a silicon lens (diameter of $241 \mu \mathrm{m}$, height of $4.38 \mu \mathrm{m}$ ) observed in reflection; Fig. 5(b) presents a profile extracted from Fig. 5(a), showing an average roughness of $\mathrm{Ra}=4.2 \mathrm{~nm}$ and a peak-to-valley roughness of $\mathrm{Rt}=26.7 \mathrm{~nm}$. The representation in Fig. 4(b) is equivalent to the result provided by a Twyman-Green or a Mach-Zehnder interferometer working with an objective illumination lens producing a spherical wave and positioned in a confocal arrangement with the test lens. With DHM, passing from one imaging mode to the other is a straightforward and purely software operation that can be performed on a single hologram without any change in the experimental arrangement. The deviation from the ideal sphere is given in the paraxial or thin lens approximation as a result of the parabolic model used to calculate the digital phase mask [Eq.(3)]. If desired, other mathematical models can be used to compute the digital phase mask, such as higher-order polynomial functions or Zernike polynomials.

To verify the accuracy of DHM, measurements on a silicon microlens array obtained with DHM are compared with a reference measurement performed on the same sample by SUSS MicroOptics SA with a WLI. The lens diameter is $241 \mu \mathrm{m}$ and the lens height is $4.38 \mu \mathrm{m}$. The ROC obtained with both techniques on the same ten lenses is compared. The measurements are in good agreement: The average ROC measurement is $1643 \pm 5 \mu \mathrm{m}$ for DHM and $1632 \pm 2 \mu \mathrm{m}$ for the WLI. The difference between the measurements is therefore less than $1 \%(0.71 \%)$.

To point out the versatility of DHM, three microoptical components of different shapes were investigated with the same transmission microscope without 
any modification of the system except an adaptation of the FOV performed by simply changing the microscope objective. The phase perspective images of these lenses are presented in Fig. 6 . The lenses under investigation were a cylindrical quartz refractive transmission lens [Fig. 6(a); diameter of $160 \mu \mathrm{m}$, maximal measured height of $7.73 \mu \mathrm{m}$, measured ROC of $417.8 \mu \mathrm{m}$ ], a quartz refractive transmission lens [Fig. 6(b); diameter of $240 \mu \mathrm{m}$, maximal measured height of $21.15 \mu \mathrm{m}$, measured ROC of $351 \mu \mathrm{m}]$, and a square quartz lens array [Fig. 6(c); pitch of $500 \mu \mathrm{m}$, maximal height of $5.5 \mu \mathrm{m}$, ROC of $5600 \mu \mathrm{m}$ ). This illustrates that DHM is definitely not limited only to spherical lenses and that no important modification of the setup or careful adjustment of the sample is required.

\section{Conclusion}

This paper has illustrated some of the possibilities offered by DHM technology in micro-optics testing. The digital reconstruction process involved in DHM makes it a versatile tool for obtaining rapidly from a single hologram a wide range of information on microlenses such as surface topography, diffracted wavefront, phase function, aberrations, ROC, lens height, and surface roughness.

Compared to classical PSI, DHM offers similar performance in terms of resolution, precision, repeatability, and FOV but can be considered as an attractive solution as a result of five main features:

- The acquisition rate is higher because a complete description of the complex wavefront is obtained from a single hologram.

- The sensitivity to external perturbations (vibration and ambient light) is reduced since the capture time can be reduced to a few tens of microseconds.

- The accuracy is not intrinsically limited by the precision of the control of moving parts, such as piezoelectric transducers.

- A DHM instrument can be used without adaptations to investigate a wide variety of micro-optical component shapes, including cylindrical, square, and strongly aspheric lenses.

- It is easy to use and flexible for implementations in automated processes for quality control because of the robustness of the technique regarding positioning tolerances.

This research was supported by Swiss National Science Foundation grant 205320-103885 and by CTI grants 6606.2 and 7152.1. Applications to the shape control of micro-optics were carried out with the help of the projet DISCO (Distance Shape Control) di- rected by the Bremer Institut für Angewandte Strahltechnik.

\section{References}

1. J. W. Goodman and R. W. Lawrence, "Digital image formation from electronically detected holograms,” Appl. Phys. Lett. 11, 77-79 (1967).

2. M. A. Kronrod, N. S. Merzlyakov, and L. P. Yaroslavskii, "Reconstruction of a hologram with a computer," Sov. Phys. Tech. Phys. 17, 333-334 (1972).

3. U. Schnars and W. Jüptner, "Direct recording of holograms by a CCD target and numerical reconstruction," Appl. Opt. 33, 179-181 (1994).

4. E. Cuche, P. Marquet, and C. Depeursinge, "Simultaneous amplitude and quantitative phase-contrast microscopy by numerical reconstruction of Fresnel off-axis holograms," Appl. Opt. 38, 6994-7001 (1999).

5. M. K. Kim, "Tomographic three-dimensional imaging of a biological specimen using wavelength-scanning digital interference holography," Opt. Express 7, 305-310 (2000).

6. T. Colomb, E. Cuche, F. Montfort, P. Marquet, and C. Depeursinge, "Jones vector imaging by use of digital holography: simulation and experimentation," Opt. Commun. 231, 137-147 (2004).

7. N. Demoli, D. Vukicevic, and M. Torzynski, "Dynamic digital holographic interferometry with three wavelengths," Opt. Express 11, 767-774 (2003).

8. V. Kebbel, J. Muller, and W. P. O. Juptner, "Characterization of aspherical micro-optics using digital holography: improvement of accuracy," in Interferometry XI: Applications, Proc. SPIE 4778, 188-197 (2002).

9. K. O. Mersereau, C. R. Nijander, A. Y. Feldblum, and W. P. Towsend, "Testing and measurement of microlenses," in Miniature and Micro-optics and Micromechanics, Proc. SPIE 1992, 210-215 (1993).

10. J. Schwider, S. Haselbeck, H. Schreiber, H. Sickinger, O. Falkenstorrer, N. Lindlein, T. Keinonen, S. Sheridan, and N. Strwubl, "Production and control of refractive and diffractive microlenses," in Miniature and Micro-Optics and Micromechanics, Proc. SPIE 1992, 102-113 (1993).

11. P. Hariharan, B. F. Oreb, and T. Eiju, "Digital phase-shifting interferometry: a simple error-compensating phase calculation algorithm," Appl. Opt. 26, 2504-2506 (1987).

12. K. J. Weible, R. Volkel, M. Eisner, S. Hoffmann, T. Scharf, and H. P. Herzig, "Metrology of refractive microlens arrays," in Optical Micro- and Nanometrology in Manufacturing Technology, Proc. SPIE 5458, 43-51 (2004).

13. E. Cuche, P. Marquet, and C. Depeursinge, "Spatial filtering for zero-order and twin-image elimination in digital off-axis holography," Appl. Opt. 39, 4070-4075 (2000).

14. E. Cuche, P. Marquet, and C. Depeursinge, "Aperture apodization using cubic spline interpolation: application in digital holographic microscopy," Opt. Commun. 182, 59-69 (2000).

15. H. Takajo and T. Takahashi, "Noniterative method for obtaining the exact solution for the normal equation in least-squares phase estimation from the phase difference," J. Opt. Soc. Am. A 5, 1818-1827 (1988). 